Chapter 11

\title{
Review of Wireless Brain-Computer Interface Systems
}

\author{
Seungchan Lee, Younghak Shin, Soogil Woo, \\ Kiseon Kim and Heung-No Lee \\ Additional information is available at the end of the chapter \\ http://dx.doi.org/10.5772/56436
}

\section{Introduction}

Over the past two decades, the study of the Brain Computer Interfaces (BCI) has grown dramatically. According to Scopus search engine, a search result with the keyword "Brain Computer Interface" returns only two papers for year 1991. But, the same query returns 897 journals and conference papers for year 2011 (date of search: March 14, 2013). BCI systems provide a new communication channel to humans who use it. They measure neurophysiological signals of the human, electroencephalogram (EEG) in particular. EEG based BCI systems are designed to decode the intension of the human user and generate commands to control external devices or computer applications. The human can produce these commands by generating the neurophysiological signals intentionally. This process can become more successful - fast and accurate - through training and practice. This technology allows the users with new experiences which enable a direct communication between the human and the computers or external devices such as home appliances, and prosthetic devices.

The BCI systems consist of two parts, signal acquisition and translation (see page 7 Figure 2). The signal acquisition part contains electrodes, analog circuit and digital system for neurophysiological signal recording and transmission. The translation part is normally computing devices which are equipped with high performance processor such as laptops, PDAs, and smart phones. With an application program, this part performs algorithmic processes such as feature extraction and classification to convert the raw neurophysiological signals into computer readable messages. Depending on the type of connection between the two parts, we can divide BCI systems into two kinds, wired versus wireless BCI systems.

Many conventional BCI systems are wired. With just three electrodes positioned the occipital lobe, the acquisition part of wired BCI systems generally comes with bulky and heavy amplifiers and preprocessing units. Connection wiring is usually complicated with a large 
number of cables between the electrodes and the acquisition part. For these reasons, preparation time for measuring EEG signals is typically very long. In addition, user's movement is limited due to cable constraints. Therefore, the application of BCI systems is difficult to escape from laboratory scale experiments. These restrictions make the types of applications for which BCI systems can be made useful be severely limited. Wireless BCI systems are to eliminate the wire connection, between the signal acquisition and the translation part, with the use of a wireless transmission unit such as Bluetooth and Zigbee modules. Removing wire connections, portability of BCI systems is greatly improved. Postures and movements of users wearing the acquisition part of wireless BCI systems are also unimpeded. These desirable aspects of wireless BCI systems promote to go beyond a laboratory scale experiments and to develop everyday-life applications.

With portable wireless BCI systems, various real-life applications are under development now. In the early days of BCI researches, cursor control and speller applications were developed mainly targeted for helping the disabled people. Recently, with growing interest, wireless BCI systems have been applied in entertainments as well. For example, Emotiv and Neurosky companies have recently released their wireless BCI headsets for entertainment uses such as brain gaming and mind monitoring. Moreover, international research groups have applied wireless BCI systems for interesting new applications such as home automation system based on monitoring human physiological states [29], cellular phone dialing [28], and drowsiness detection for drivers [19][20][32].

In this book chapter, we will review recent research trends in wireless BCI systems. In Section 2, we summarize several research topics in wireless BCI systems such as electrodes, embedded systems, user-friendly designs, and novel applications. We then take a closer look into emerging wireless BCI systems designed by BCI researchers, and discuss general BCI systems recently introduced into the market in Section 3. In Section 4, we discuss current challenges and possible future research directions on wireless BCI systems. Finally, we provide concluding remarks in Section 5.

\section{Research trends of wireless BCI systems}

Wireless brain computer interface (BCI) systems are neurophysiological signal acquisition and processing systems where acquired physiological signals are wirelessly transmitted to the translation unit. Wireless systems, unlike their traditional wired counterparts, are designed to provide convenience in monitoring the neurophysiological signals of users.

Compared to conventional wired BCI systems, wireless BCI systems provide enhanced portability and wearability, facilitated by elimination of the wire connections between the wearable acquisition unit and the translation unit. The translation unit is usually housed in a portable device such as laptops and smart phones. This improvement provides an easy installation process and freedom of postures for users. Furthermore, owing to advanced integrated circuit designs, the components of the wireless BCI systems are small in sizes and efficient in power consumption. Employing these components, the acquisition part of wire- 
less BCI systems can be miniaturized. These advantages allow wireless BCI systems to be shaped in user friendly styles such as baseball caps [14][19][21][29], headsets [16][17][23][25] [27], and headbands [18][20][22][28][30][32]; thus, applying them in various applications such as entertainments and health care becomes easier than ever before.

Even though wireless BCI systems may provide a number of advantages, there are still many issues that need to be resolved including improving signal quality, more compact and stylish system designs, and excavation of useful applications. First, the quality of the measured EEG signals has to be improved for more precise classification of user's intensions. The measured EEG signals are easily contaminated by various noise sources such as the presence of other physiological signals and the power line noise. Moreover, various impediments exist in electrodes-scalp interfaces such as hairs, sweat, and stratum corneum of skin [6]. These obstacles cause deleterious effects in signal measurements with high leakage currents and high contact impedances. Due to these difficulties, EEG signals get easily corrupted and the quality of measured signals is often undesirable. Consequentially, these factors lead to drop of application accuracy. Second, stylish, miniaturized and light weight wireless $\mathrm{BCI}$ systems are necessary for daily life application and long-term wearing. Conventional wired BCI systems are bulky, not user-friendly in system appearances because of their complicated connection between electrodes and signal acquisition part. Multi-channel electrode installation is also inconvenient and time-consuming, usually taking more than 30 minutes. Thus, the users are easily irritated and long-term monitoring becomes difficult. Third, killer applications are needed. Many researchers and developers introduced various applications related to entertainments and health care. But such contributions are still not enough to make any fundamental change in our life.

To help these issues, research groups are paying attention to the following aspects:

1. Advanced electrodes for measuring clean EEG signals

2. Low-power, miniaturized, portable and wearable BCI systems

3. A killer application, an application of such a great value and popularity that it assures the success of the BCI technology.

In this section, we aim to analyze the current status of research and development efforts in these directions.

\subsection{New electrodes for EEG signal acquisition}

Among the above research topics, the development of advanced EEG electrodes which measure brain signals precisely with low noise is the most important challenge. Practically, the signal acquisition part of general wireless BCI systems only contains a signal acquisition circuit and a micro-processor based embedded system for transmission of the measured EEG signals. For example, a well-known Emotiv EPOC neuro-headset is composed of 14 channel electrodes and a small integrated embedded system powered by an onboard battery for signal conditioning and transmission. In the translation part of wireless BCI systems, analysis of acquired signals is performed either online or offline on a computer or a mobile 
device which is equipped with high-performance processors. For this segregated structure to work well, signal acquisition part of a wireless BCI system should be devoted to high fidelity signal recording. To provide clear EEG signal acquisition at the electrode-skin interface, development of outstanding electrodes becomes a critical issue. For this reason, many research groups have recently been interested in developing advanced electrodes which can provide low-noise recording, convenience in installation, and comfort even in long-term wearing.

In conventional wired BCI systems, passive electrodes are widely used to measure EEG signals. Generally, these electrodes are disc or ring shaped and are made of $\mathrm{Ag} / \mathrm{AgCl}$ alloy [10]. Due to their simple structure, it is easy to make them small. However, they have many disadvantages as well. Extra treatments are essential for recording reliable EEG signals because the scalp potentials are only on the order of several micro-volts and thus very noise-sensitive. Treatments are needed, including a hair preparation step and the use of conductive gels or glues for better attachment and higher conductivity. These preparations induce discomfort and require long preparation time. Furthermore, the conductive gels easily desiccate and lose their adhesion. These problems bring about worse contact impedances at electrode-scalp interfaces, causing a large reduction of signal-to-noise ratio. In addition, the quality of recorded signals is sensitive to cable vibrations [17]. For these reasons, the longterm monitoring of EEG signals using passive electrodes is not feasible.

Recently, to overcome the weaknesses of passive electrodes, many researchers have studied advanced electrodes. For examples, research of dry electrodes is active recently. Generally, dry electrodes are defined as those that do not require the use of conductive gels or glues for installation process. Thus, a user can conveniently attach the electrodes to the user's scalp without any hair arrangement. To make dry contact at the electrode-skin interface, researchers employ special materials or shapes in the design of dry electrodes. Extensive research has produced a huge variety of electrode materials and structures, including conductive rubber [8][9], conductive carbon nanotubes [7], micro-tip structures [6], micro-machined structures [14][15][20], non-contact types [4][5], spring-loaded fingers [2][13], bristle structures [3], and conductive foams [27]. The most widely used dry electrode design is a set of contact posts which look like fingers [13][16]. This design has an advantage in contact ability because it is easy to penetrate into the scalp through the hair without an extended hair arrangement. Recently, some research groups have altered this finger design to produce advanced mechanical designs, such as spring-loaded fingers [2] or bristle structures [3]. These designs seem to provide flexibility and geometric adaptation between the sensor and the irregular scalp surface to obtain low interface impedance. To achieve low contact impedance and provide a robust and stable electrical interface, some research groups have employed multi-walled carbon nanotube arrays [7] and micro-tip structures [6], which are able to penetrate the outer skin layer (which is 5 to10 um thick and called the stratum corneum).

While we can reduce the installation time significantly using dry electrodes, the contact impedance between the scalp and the electrodes is higher than that with gel-based passive electrodes due to the absence of conductive gels. Thus, signal quality of dry electrodes would be not better than that of the gel-based passive electrodes. To overcome this draw- 


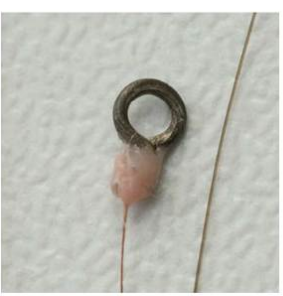

a

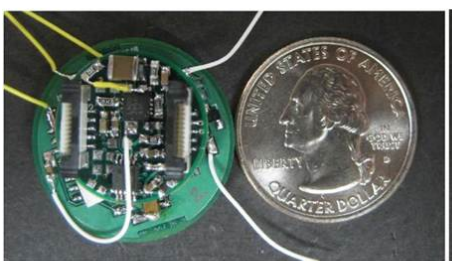

e

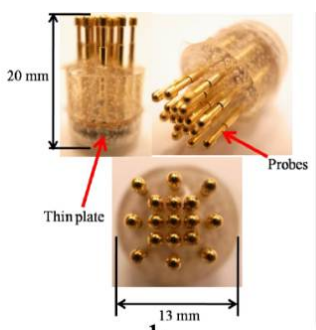

$b^{\text {Bint }}$

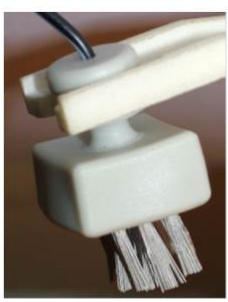

$\mathrm{c}$

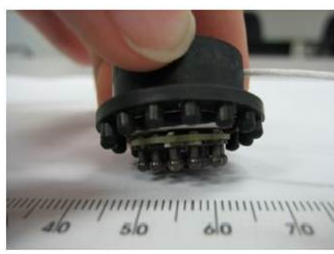

d

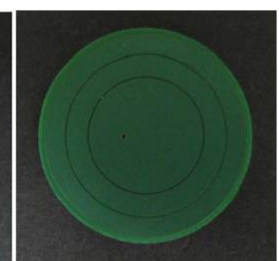

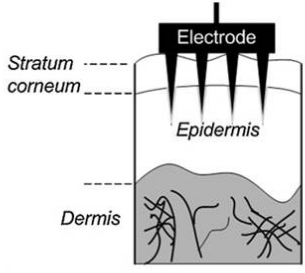

$\mathrm{f}$

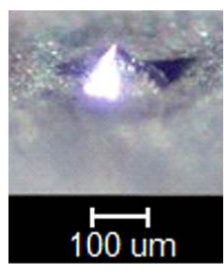

Figure 1. Various EEG electrode types. (a) a miniature passive ring electrode [10] (b) a spring-loaded dry electrode [2] (c) a bristle-type dry electrode [3] (d) the Quasar hybrid EEG biosensor [11] (e) a non-contact-type active dry EEG sensor [4] (f) Diagram of a micro-tip electrode and the pyramidal shape of a micro-tip [6].

back, many research groups have been interested in active electrodes. Active electrodes contain amplifier or buffer circuits integrated to the electrodes themselves [1][4][5][7][11][12] [13]. This amplifier or buffer circuits are located between the electrodes and the signal acquisition frontend. They are aimed at impedance conversion. Providing high input impedance on the electrode-amplifier interface, active circuits reduce the distortion of the measured signals. This is desired for dry electrodes which do not use conductive fluids. Also, the low output impedance of the amplifier eliminates artifacts caused by posture changes in mobile environments. Therefore, the quality of measured physiological signals can be remained in a desirable state by the use of active electrodes.

Recent wireless BCI systems are equipped with active dry electrodes to combine the advantages of active and dry electrodes such as convenient installation and high fidelity signals. Because these electrodes provide more robust and stable signal quality in mobile environments, they are suitable for wireless BCI systems. Researchers working on the development of advanced electrodes have produced a variety of active dry electrodes. Valchinov et al. designed body surface electrodes equipped with a biopotential amplifier using two op-amps [1]. Matthews et al. designed an ambulatory wireless EEG system using Quasar hybrid biosensors [11][12][13]. In this type of sensors, they employ a special circuit which uses the common mode follower (CMF) technology. This technology provides an ultra-high input impedance to ensure low distortion of the biopotential signals. Chi et al. designed and built dry and non-contact electrodes [5]. In their dry electrodes, a unity gain buffer is used to reduce the effects of cable artifacts and external interference. Their non-contact electrodes also 
integrate discrete circuits to achieve high input impedance. To further optimize the size and power consumption of such electrodes, some researchers have employed customized ASIC designs for amplifiers. Xu et al. produce a low-power 8-channel active electrode system [17]. In this system, to reduce the power consumption of voltage buffers in dry electrodes, they designed active electrodes including ASICs based on chopper instrumentation amplifiers.

\subsection{Wireless BCI system design and structure}

In EEG-based wireless BCI systems, additional signal conditioning is essential to enable the transmission of precise neurophysiological signals. Many noise sources are present such as physiological interferences and power line noise. Physiological interferences are the other biopotential signals such as electromyogram (EMG), electrocardiogram (ECG), and electrooculogram (EOG). They have relatively lager amplitudes around $50 \mathrm{uV}$ and up to $20-30 \mathrm{mV}$ while the amplitude of EEG signals is much smaller on the scale of roughly 10 100uV. Thus, the EEG signals are easily buried by these physiological signals unavoidably. In the case that the BCI system is connected to a desktop which operate with the electric power outlet, we also have to consider the power line noise as well. The power line noise contaminates the desired EEG signals in the range of 50 or $60 \mathrm{~Hz}$. Furthermore, the users of portable wireless $\mathrm{BCI}$ systems are usually in an active state making free motions and postures, whereas the users of wired BCI system are asked to stay in a motionless state, while their EEG signals are monitored. Therefore, the measured EEG signals of wireless BCI systems are also subject to heavy motion and vibration artifacts.

To avoid interference from the various noise components and recognize the user's intention correctly, the system must be designed carefully. Figure 2 is the general block diagram of a typical wireless BCI system. In EEG acquisition block of the system, there are two main parts, namely, the analog front end circuit and the digital system.

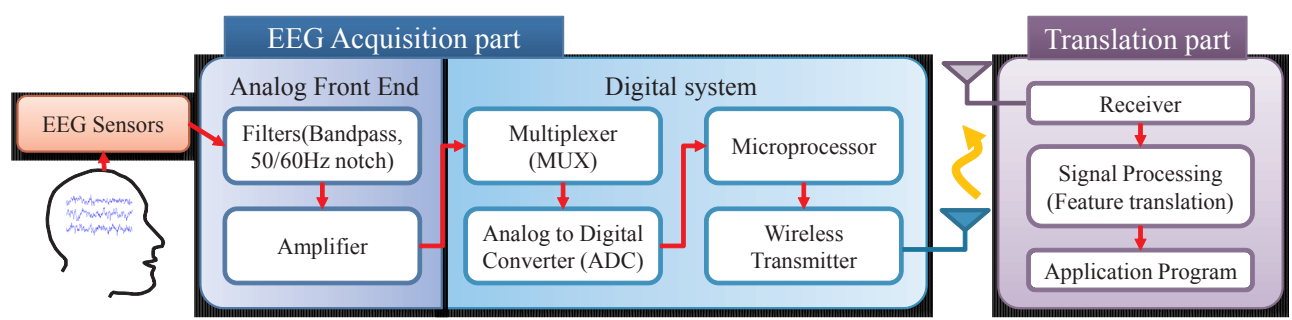

Figure 2. Block diagram of a typical wireless $\mathrm{BCl}$ system

In the analog front-end stage, the amplifier and bandwidth limiter circuits are included to make more robust and reliable EEG signals from the sensitive raw signals. Because the amplitude of EEG signals is quiet small, the pre-amplification of the measured EEG signals at the analog front end is extremely important. In this amplification process, many developed wireless BCI systems use operational amplifiers or instrumentation amplifiers. Those amplifiers normally provide a gain ranging from thousands to hundreds of thousands. Amplifica- 
tion with high gain provides greater robustness against a variety of noise sources. However, we need to determine a suitable amplification gain to maximize the signal resolution in the analog digital converter (ADC) because the ADC has a restricted input dynamic range. Therefore, the amplification gain of the analog front end varies depending on the components of the digital system.

We also need a frequency filtering procedure to remove various noise components. The EEG signals occupy a narrow bandwidth: normally from $0.1 \mathrm{~Hz}$ to less than $50 \mathrm{~Hz}$. Thus, filtering is helpful for extracting useful signals from the desired frequency bands. To filter out signals from useless frequency bands, the analog front-end of the system takes both a low-pass filter and a high-pass filter. Especially to filter out the power line noise, a notch filter which eliminates the specific frequency components of signals is also applied in this stage. Those filtering processes are performed using passive or active filtering circuits [24][26].

In the digital system stage, four integrated circuits are included: a multiplexer, an ADC, a microprocessor, and a wireless transmission unit. Generally, most EEG-based wireless BCI systems support multi-channel recording. To measure multi-channel signals simultaneously, a multiplexer is needed to access all of the channels. Because the measured EEG signals are analog signals, an ADC has to be included to process the recorded EEG data on the digital circuits. This integrated circuit transforms the EEG analog signals into discrete digitized data with a specific sampling frequency. The sampling frequency is determined by the speed of the microprocessor, wireless transmission, and translated frequencies of EEG features. Formally, researchers and system developers choose the sampling frequency between about $100 \mathrm{~Hz}$ and $1000 \mathrm{~Hz}$. The microprocessor makes data packets from the corrected EEG data and hands them over to the wireless transmission unit. The microprocessor also manages the components of the entire system. Some wireless BCI systems load the feature extraction algorithm on the microprocessor to process the EEG signals internally [19][20][21] [29][30]. Because the recorded multichannel EEG data is transmitted from the portable EEG acquisition device to the host system, the wireless transmission unit is essential. Regarding the protocol of wireless transmissions, various communication modules are employed for transmission of the measured signals from the signal acquisition unit to the translation unit, such as Bluetooth and IEEE 802.15.4 Zigbee. Bluetooth has many advantages such as sufficient transmission rates and wide accessibility. Thus, many wireless BCI systems employ this transmission module. Including analog front-end and digital system stage, the acquisition unit of wireless BCI systems generally operates onboard power sources such as Li-ion, Li-polymer, and NiMH batteries.

Because the analog front-end and digital system parts have to be loaded in portable and wearable acquisition part of wireless BCI systems, longer operation time and small size are necessary in system specifications. Thus, system developers should choose low-power components with smaller packages. Recently, many semiconductor manufacturers have released low-power microprocessors and integrated analog front end circuits for bio-potential measurements. For example, Texas Instruments released the ADS129x series integrated circuit solutions [34] for the analog front end of ECG/EEG applications. This series provides up to 8-channel high-resolution ADCs and a built-in programmable gain amplifier (PGA) with 
low noise and low power consumption features. In the microprocessor area, various ultralow power processors have been released on the market for portable devices. The most widely used microprocessors are the PIC24 microcontroller series [35], the dsPIC digital signal controller series [36] (manufactured by Microchip Technology) and the MSP430 microcontroller series [37] (manufactured by Texas Instruments). In particular, the dsPIC processor series is applied as the processing unit of the Emotiv EPOC system [38].

Regarding the design approach of system appearance, a variety of designs have been adopted depending on the application purpose and target users. Widely used styles of the acquisition part of wireless BCI systems include headsets [16][17][23][25][27], head bands [18][20] [22][28][30][32], baseball caps [14][19][21][29], and military helmets [13]. In designing the appearance of wireless BCI systems, we need to consider several factors, such as wearability, stability, and convenience of installation. To provide long-term monitoring capability, wearable part of wireless BCI systems has to be light with comfort fitting. Also, convenient installation is necessary to save time in the set up process. Appropriate pressures are also needed to maintain stable electrode positions and low impedance characteristics at the sensor-skin interfaces. Additionally, to allow for the diversity of users' head sizes, the materials used in wireless BCI systems should be flexible, or size adjusters must be added. Various designs of wireless BCI systems are shown in Figure 3.

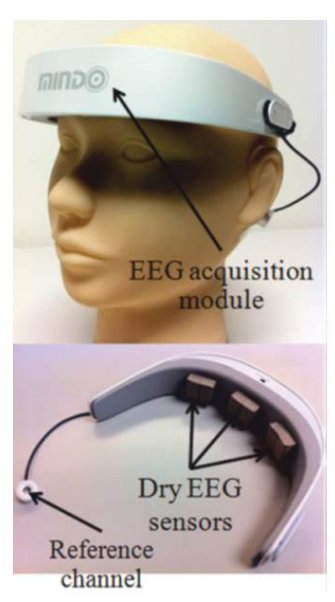

a

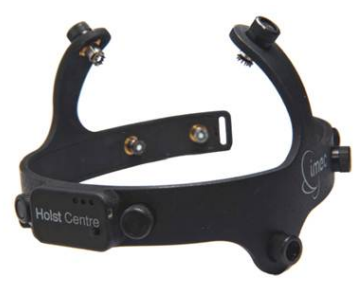

$\mathrm{b}$

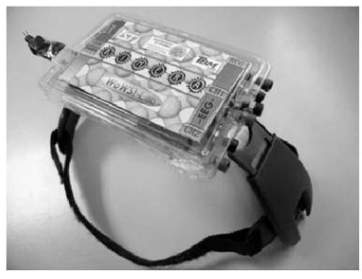

d

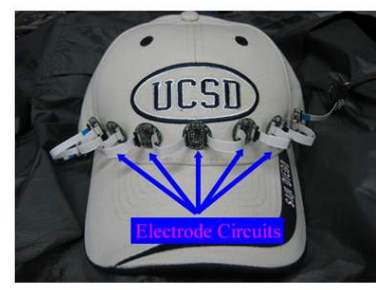

c

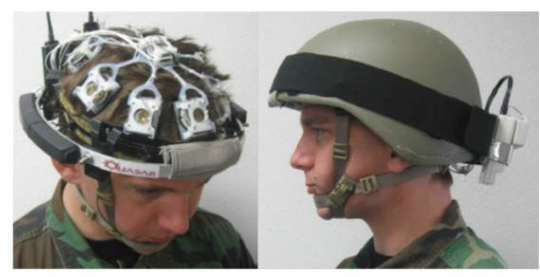

e

Figure 3. Various designs of wireless BCI systems: (a) wearable EEG acquisition headset [27], (b) 8-channel EEG monitoring headset [16], (c) baseball cap-based EEG acquisition device [14], (d) wireless EEG system for SSVEP application [25], and (e) soldier's Kevlar helmet-based ambulatory wireless EEG system for real-time workload classification [13]. 


\subsection{Signal features and applications}

In EEG-based BCI systems, they translate the specific signal features that reflect the user's intentions or cognitive states into commands or feedback signals for controlling of the target applications. For these operations, BCI systems analyze and capture the user's intensions based on detection of ERPs [33] or power spectra changes in specific brain rhythms.

Most research groups have focused on sensorimotor rhythms (SMRs) [39] and event related potentials (ERPs) [40], including visual evoked potentials (VEPs) such as P300 [41] and steady-state visual evoked potentials (SSVEP) [42]. The SMRs are spontaneous responses which can be actively generated by motor imageries, such as the left hand, the right hand, or the foot movements measured by electrodes placed on the scalp over the sensorimotor cortex area. These rhythms appear as suppression or enhancement of the power spectra, called event-related desynchronization (ERD) and event-related synchronization (ERS). The VEPs are behavioral responses which are passively synchronized by the frequency of flickering visual stimulus from the occipital lobe. Because the SMRs and VEPs reflect the user's intensions, we can utilize them as a means to control commands in applications. Moreover, various cognitive states are also studied, such as drowsiness, alertness, and mental focusing [27] [29][30][32]. These cognitive states are related to the power changes of specific rhythms, called alpha, beta, theta rhythms and so on. Several studies have shown that the power of the alpha rhythm has a negative relationship with mental concentration [27][29][30]. Also, researchers have found that when subjects feel sleepy or fall into a deep sleep, the power spectra of alpha and theta bands change depending on these drowsiness conditions [29][30] [32]. Using these relationships, the BCI systems detect the user's cognitive states and provide feedbacks such as focusing indicator [27] and sleep warning [32].

Among the signal features mentioned above, many researchers have chosen VEPs or users' cognitive states as a means for BCI based controls. The reason is that these features are easy to generate and provide good accuracy in the application of BCI systems. Also, the features of VEPs and cognitive state make it easy to classify users' intentions with a relatively simple feature extraction method, and a small number of electrodes and training session are needed to achieve higher accuracy. Compared with ERPs like VEPs, the SMRs can be generated by voluntary imagination, such as motor imagery, generally require long-term training to achieve higher accuracy in BCI applications. Furthermore, approximately $30 \%$ of normal people cannot generate SMRs due to the phenomenon of BCI illiteracy [43].

In the application parts of BCI systems a variety of promising rehabilitation-related applications have already been developed with EEG-based wired BCI systems. Because BCI systems measure and analyze neurophysiological signals, these systems provide practical assistance for patient diagnosis, treatment, and rehabilitation. For example, using BCI systems, long-term monitoring of EEG signals assists the diagnosis of epilepsy and the prediction of epileptic seizures [44]. For people with severe motor disabilities, the P300 speller [41] and wheelchair control [45] applications provide practical assistance in everyday life by providing non-muscular motor functions. 
In spite of these useful applications, the dissemination of BCI systems is limited because of the drawbacks of wired BCI systems. Wired BCI systems are generally bulky, complicated, and expensive. Also, the users of wired BCI systems are confined to a limited space without freedom of postures and movements. To overcome these limitations, wired BCI systems are gradually being replaced with the wireless BCI systems.

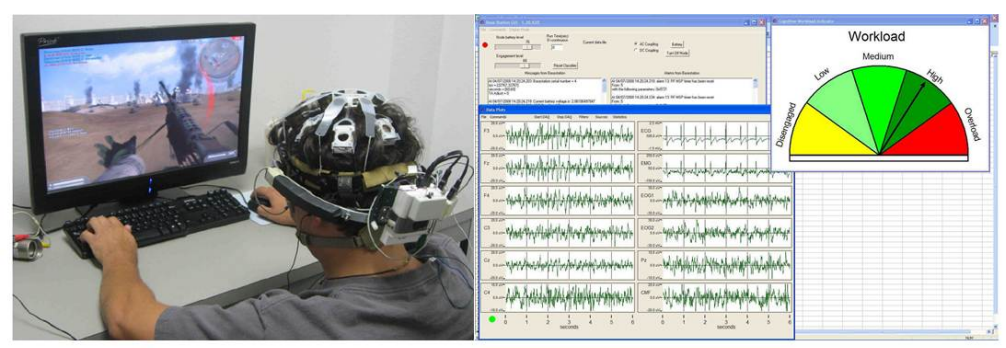

a

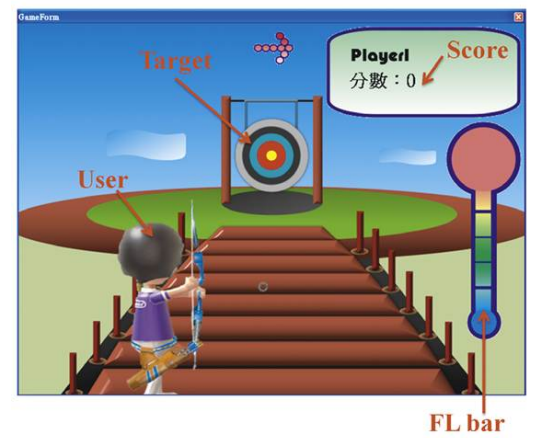

b

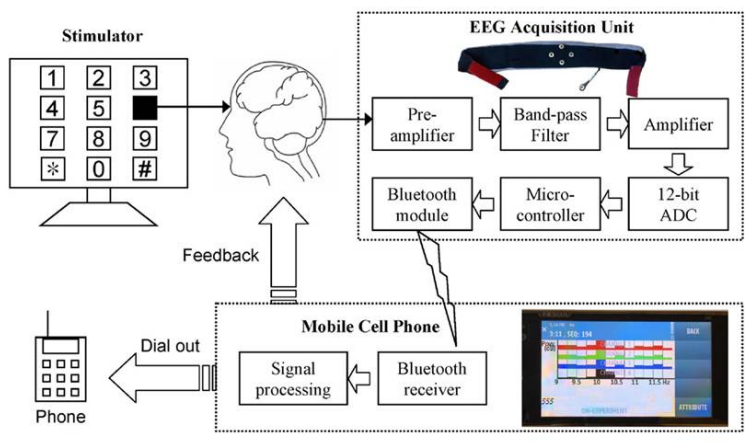

c

Figure 4. Applications of wireless BCl systems: (a) workload classification application screenshot (data collection and engagement classifier running on the gaming subject) [13], (b) EEG-based BCl archery game screenshot [27], and (c) SSVEP-based dialing application using smart phone [28].

Recently, with the development of wireless BCI systems, researchers have shifted their focus from applications for disabled people to applications of interest to the general public in the entertainment, smart living environment, and cognitive neuroscience areas. In the entertainment area, Liao et al. developed an EEG-based gaming interface based on a real-time focusing detection algorithm with a wireless EEG acquisition device [27]. For smart living environment, Lin et al. developed an environmental auto-control system based on human physiological states, such as drowsiness and alertness [29][30]. Similarly, Guge et al. developed a smart home control system based on P300 EEG response [31]. For mobile applications, Wang et al. developed a cellular phone dialing application [28]. In cognitive state monitoring, D'Arcy et al. developed a diagnostic device which provides an evaluation of an individual's conscious awareness based on various ERP components [33]. In this research, 
they found that sensation, perception, attention, memory, and language are properly related with the P1, mismatch negativity (MMN), P300 (tones), P300 (speech), and N400 responses. Also, Matthews et al. developed a real-time workload classification system during subject motion with a compact ambulatory wireless EEG system [13]. Figure 4 shows application examples of wireless BCI systems.

\section{Review of wireless BCI systems}

Over the past 20 years, many research achievements associated with BCI and the neurosciences have been made and they have helped stimulate the interest of the general public. Owing to the advances in wireless BCI systems, bulky wired biopotential acquisition systems have been replaced with portable and wearable devices. Following this trend, the number of published papers with the topic of wireless BCI systems and (their) applications is being continuously increased. A few commercial companies have developed and released portable wireless EEG acquisition systems with interesting new entertainment applications. Now, measuring brain activity is no longer limited to hospital-based medical diagnostics, but includes more courageous applications aiming at changing the lifestyle of users. In this section, we aim to first review several wireless BCI systems which have appeared in recent research articles. Second, we will introduce several examples of wireless BCI systems which have been lately released into the market for consumer and research usages.

\subsection{Wireless BCI systems in scientific papers}

In the research field, many research articles have been published in the last decade with the topic of wireless BCI systems. There are some distinct features in them such as the use of dry electrodes and novel applications which we are interested in reviewing in this subsection. For example, see the wireless BCI systems listed in Table 1. This table shows system specifications such as the number of channels and operation hours. They are all wireless and wearable systems, some aiming for applications that average people can find useful, including drowsiness detection and workload monitoring. Specifications for each system are optimized for its own target application.

In what follows, we will briefly review each of the system listed in Table 1

\subsubsection{Wireless BCI system for archery game control}

Recently, utilizing wireless BCI systems, the number of game applications has been increased. For example, Liao et al. [27] have developed an EEG based BCI device for an archery game control. This device is designed as a user friendly headset (see Figure 3 (a)) and equipped with three channels, each channel with a dry EEG sensor. For the control of archery game, the sensors measure the power of alpha rhythm collected off of user's forehead when a user concentrates on a target. This power value is converted to a measure of focusing intensity in real time. Any user can test out the level of one's concentration effort using 


\begin{tabular}{|c|c|c|c|c|c|c|}
\hline Reference & $\begin{array}{l}\text { Liao et al., } 2012 \\
\text { [27] }\end{array}$ & $\begin{array}{l}\text { Yu Mike Chi et } \\
\text { al., } 2012 \text { [5] }\end{array}$ & $\begin{array}{c}\text { Nuno Sérgio } \\
\text { Diasa et al., } 2012 \\
{[6]}\end{array}$ & $\begin{array}{l}\text { Chin-Teng Lin et } \\
\text { al., } 2010 \text { [20] }\end{array}$ & $\begin{array}{l}\text { R. Matthews et } \\
\text { al., } 2008 \text { [13] }\end{array}$ & $\begin{array}{l}\text { Lindsay Brown et } \\
\text { al., } 2010 \text { [16] }\end{array}$ \\
\hline Signal features & $\begin{array}{l}\text { Mental focusing } \\
\text { feature }\end{array}$ & SSVEP & Not mentioned & $\begin{array}{l}\text { Alpha and Theta } \\
\text { rhythms }\end{array}$ & $\begin{array}{l}\text { Cognitive state } \\
\text { (workload) }\end{array}$ & Not mentioned \\
\hline Application & $\begin{array}{c}\text { Archery game } \\
\text { control }\end{array}$ & Neuro-feedback & Not mentioned & $\begin{array}{l}\text { Drowsiness } \\
\text { detection }\end{array}$ & $\begin{array}{l}\text { Workload } \\
\text { monitoring }\end{array}$ & Not mentioned \\
\hline Sensor type & $\begin{array}{l}\text { Dry foam-based } \\
\text { EEG sensor }\end{array}$ & $\begin{array}{c}\text { Dry and } \\
\text { noncontact } \\
\text { electrodes }\end{array}$ & $\begin{array}{l}\text { Micro tip dry } \\
\text { electrodes }\end{array}$ & Not mentioned & $\begin{array}{c}\text { Finger type } \\
\text { hybrid biosensor }\end{array}$ & $\begin{array}{c}\text { Dry electrode } \\
\text { with contact post }\end{array}$ \\
\hline \# of channels & 3 channels & Not mentioned & 5 channels & 3 channels & $\begin{array}{c}7 \text { channels EEG, } \\
\text { ECG, EMG, EOG } 2 \\
\text { channels }\end{array}$ & 8 channels \\
\hline $\begin{array}{c}\text { Frontend } \\
\text { processing unit }\end{array}$ & TI MSP430 & Microchip PIC24F & $\begin{array}{c}\text { Atmel } \\
\text { Atmega128 }\end{array}$ & TI MSP430 & Not mentioned & $\begin{array}{l}\text { TI MSP430 and } \\
\text { ASIC }\end{array}$ \\
\hline $\begin{array}{l}\text { Backend } \\
\text { processing unit }\end{array}$ & PC & $\begin{array}{l}\text { Nokia N97 } \\
\text { cellular phone }\end{array}$ & PC & $\begin{array}{l}\text { ADSP-BF533 } \\
\text { embedded } \\
\text { processor }\end{array}$ & Laptop & Not mentioned \\
\hline $\begin{array}{c}\text { Transmission } \\
\text { protocol }\end{array}$ & $\begin{array}{l}\text { Bluetooth v2.0 } \\
\text { +EDR }\end{array}$ & Bluetooth & Bluetooth & $\begin{array}{l}\text { Bluetooth v. } \\
2.0+E D R\end{array}$ & Bluetooth & $2.4 \mathrm{GHz}$ \\
\hline $\begin{array}{l}\text { Signal resolution } \\
\text { and sampling rate }\end{array}$ & $12 \mathrm{bit}, 256 \mathrm{~Hz}$ & 24 bit & 16bit, $1 \mathrm{kHz}$ & $12 \mathrm{bit}, 512 \mathrm{~Hz}$ & $\begin{array}{c}\text { 16bit, } 240 \mathrm{~Hz} \text { for } \\
\text { EEG }\end{array}$ & $\begin{array}{c}11 \mathrm{bit}, 256 \sim \\
1024 \mathrm{~Hz}\end{array}$ \\
\hline $\begin{array}{l}\text { Power source and } \\
\text { operation time }\end{array}$ & $\begin{array}{l}23 \text { hours with a } \\
3.7 \mathrm{v} 750 \mathrm{mAh} \mathrm{Li-} \\
\text { ion battery }\end{array}$ & $\begin{array}{l}10 \text { hours with } 2 \\
\text { AAA batteries }\end{array}$ & $\begin{array}{c}25 \text { hours using } 2 \\
\text { AA batteries }\end{array}$ & $\begin{array}{c}33 \text { hours with a } \\
3.7 \mathrm{v} 1.1 \mathrm{Ah} \mathrm{Li-lon} \\
\text { battery }\end{array}$ & $\begin{array}{l}80 \text { hours with } 2 \\
\text { AAA batteries }\end{array}$ & $\begin{array}{l}30 \text { hours with a } \\
3.7 \mathrm{v} 140 \mathrm{mAh} \mathrm{Li}- \\
\text { Ion battery }\end{array}$ \\
\hline Design & $\begin{array}{l}\text { Headset with } \\
\text { elastic band }\end{array}$ & Not mentioned & Brain cap & Headband & Soldier helmet & Headset \\
\hline
\end{tabular}

Table 1. Comparison of wireless $\mathrm{BCl}$ systems in scientific articles

this game (see Figure 4 (b)). If a user maintains for a certain period a high level of concentration state, for example, the arrow will hit the center of target.

\subsubsection{Mobile BCI using dry and noncontact EEG sensors}

These days, smart phones come with high performance processors. They can be useful for wireless EEG monitoring. In [5], Yu Mike Chi et al. have developed a wireless BCI device based on smart phones. They have developed an smartphone application with a GUI interface for signal monitoring and analysis. They have tested two types of electrodes, dry and noncontact EEG sensors. Dry sensors consist of several spring-loaded electrodes, each comes with a finger post and a unity gain buffer. Noncontact electrodes employ CMOS amplifiers 
to improve their impedance performance. Both electrodes offer easy installation and good signal quality.

\subsubsection{Wireless BCI system based on dry electrodes with a needle-shape structure}

As mentioned in Section 2, novel electrode design is important part in wireless BCI systems. In [6], Nuno Sérgio Dias et al. have developed a wireless EEG acquisition system with novel dry electrodes. For the electrode design, they have employed 16 micro tip structures each looks like a needle (see Figure 1 (f)). They are designed to make direct contacts with electrolyte fluids of the inner skin layers of the scalp. Therefore, signal quality is satisfactory without the use of conductive gels. The proposed system consists of an EEG brain cap with five dry electrodes, an acquisition device which is attached to the brain cap, and a wireless base station connected with a computer. The acquisition device can operate for 25 hours with two AA batteries.

\subsubsection{Design of wireless BCI system for drowsiness detection}

Wireless BCI systems can be utilized in practical applications such as house control system [29][30][31] and drowsiness detection system [20] for drivers. In [20], Chin-Teng Lin et al. have proposed a real-time wireless EEG-based BCI system for drowsiness detection and shown usefulness in providing sleep alerts to a driver in car driving simulation. They have designed a wireless signal acquisition module and a signal processing module. The acquisition module is small enough to be embedded into a wearable headband. These modules are linked with each other via a Bluetooth connection. Therefore, it provides the advantages of mobility and long-term monitoring (more than 33 hours with a 1100-mA Li-Ion battery). Also, they have developed a real-time drowsiness detection algorithm. The algorithm detects the user's drowsiness by analyzing the theta rhythm and the alpha rhythm of the EEG signals.

\subsubsection{Ambulatory wireless BCI system for real time workload classification}

A compact, lightweight, and ultra-low power ambulatory wireless BCI system has been developed in [13]. This system consists of soldier's helmet with biosensors and a data acquisition unit (see Figure 3 (e)). They use Quasar hybrid biosensors which are equipped with dry electrodes shaped like a set of fingers (see Figure $1(\mathrm{~d})$ ). This system provides high freedom of motions with data quality as good as that of wet electrodes. They have also developed a real time classifier for determination of the cognitive workload of a user.

\subsubsection{Design of wireless EEG monitoring headset}

In [16], Lindsay Brown et al. have introduced a design of wireless EEG monitoring headset. This headset is equipped with the 8-channel dry electrodes as shown in Figure 3 (b). Each electrode is designed with contact posts which are coated with $\mathrm{Ag} / \mathrm{AgCl}$ for easy penetration into the user's hairs. Each electrode is connected to its electrode housing via a magnetic ball and a socket for tilting and vertical movement of electrodes. They have employed an 8-chan- 
nel EEG acquisition application-specific integrated circuit (ASIC) into which preamplifiers are embedded for low power consumption. This system can operate for long time over 30 hours using a $140 \mathrm{mAh}$ Li-Ion battery.

\subsection{Wireless BCI systems for consumer use}

In the market of consumer-grade wireless BCI systems, there are many commercial companies such as Emotiv, Neurosky, MyndPlay, PLX devices, and OCZ technology. These companies have competitively released their own wireless BCI systems along with various applications related to gaming, utilities, and mental-state monitoring. In this section, we review these wireless BCI devices for consumer use shown in Figure 5.

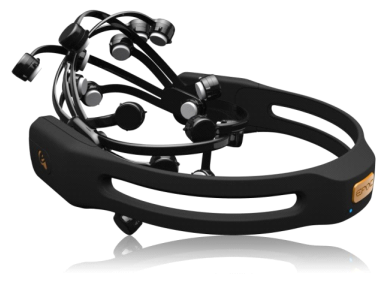

a

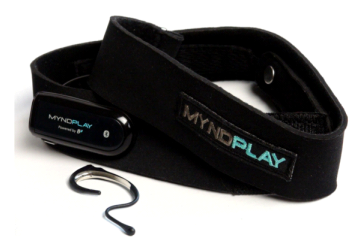

C

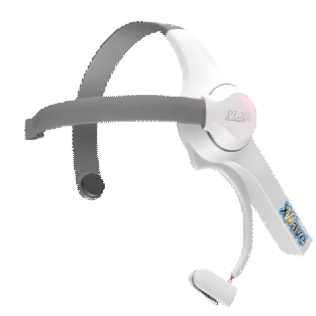

d

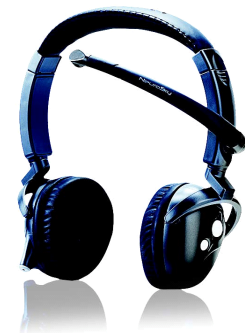

$\mathrm{b}$

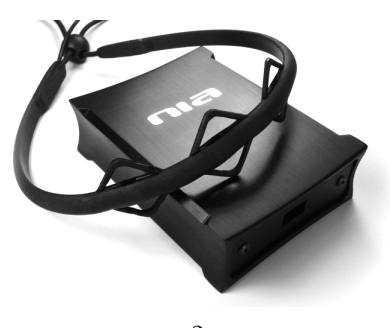

e

Figure 5. Pictures of Wireless BCI systems for consumer use: (a) Emotive EPOC headset [38], (b) NeuroSky Mind Set [46], (c) MyndPlay Brainband [50], (d) PLX devices XWave headset [49], (e) OCZ Neural Impulse Actuator [51].

The device released by Emotiv is the EPOC headset. The EPOC headset [38] is a multi-channel wireless BCI system. This headset is equipped with 14 saline-based wet-contact resistive electrodes for measuring EEG, Electrooculogram (EOG), and facial Electromyogram (EMG). Additionally, the EPOC headset also has a 2-axis gyroscope for measuring the head rotation. Employing 2.4GHz wireless connectivity, this system provides wide accessibility for devices such as PCs, laptops, and smart phones. The package of the EPOC headset provides a bundle software which contains a suite of built-in signal processing algorithms for interpretation of EEG signals. The built-in algorithms discern the user's conscious intentions, emotional states, and facial expressions based on measured EEG, Electromyogram (EMG) and Electrooculogram (EOG) signals. Through this software, the users can interact with var- 
ious applications related to virtual reality, game controlling, and brain state monitoring. These applications can be downloaded by accessing their web site.

The Neurosky Mind Set [46] is a wireless headset added with an EEG signal acquisition unit. This headset is equipped with earphones and a microphone, and a single dry-contact electrode for measuring the user's EEG signals on the user's forehead. Along with the capability of raw EEG recording, the Mind Set has the patented algorithm, named as eSense [47]. This algorithm interprets the user's mental states such as attention and meditation. These translations are estimated by monitoring the power levels in specific frequency bands such as alpha, beta and theta rhythms. The monitoring values of the brain state are utilized for making a control commands in applications.

The MyndPlay and the PLX devices are released with their own model of wireless BCI systems. MyndPlay Brainband [50] and PLX devices XWave [49] headset utilizes the ThinkGear Application-Specific Integrated Circuit (ASIC) module [48]. This module, designed by Neurosky, is a system-on-chip integrated circuit equipped with signal acquisition components. These devices come in a headset or a harness style. They have supported the control of various applications such as media player, cognitive state visualization, and arcade games for $\mathrm{PC}$ and mobile devices.

OCZ technology, a PC component manufacturer such as solid-state drives (SSD) and power supplies, has released a game controller by utilizing an wireless BCI technology. The name of this controller is Neural Impulse Actuator (NIA) [51]. Using NIA, the users can control a PC game by translating facial expressions, eye movements, and concentrated brainwave activity, instead of using traditional input devices such as a keyboard and a mouse. This device now supports various PC games including shooting, role playing, virtual worlds, and racing.

\subsection{Wireless BCI systems for research uses}

Wireless BCI systems have many advantages, such as freedom of user's postures and convenient installation. Therefore, wireless BCI systems are useful in the research field as well. Some companies have been involved in research with universities and research institutes to develop wireless BCI systems for research uses. They include, but not limited to, Advanced Brain Monitoring, Quasar, Starlab and Guger technologies (G.tec). In this subsection, we review their wireless BCI systems, as shown in Figure 6.

Advanced Brain Monitoring has recently released the B-Alert X series wireless EEG systems [52] for mobile neurophysiological data acquisition and analysis. These systems include three models that have different numbers of channels, i.e., 4, 10, and 24. Among these models, the B-Alert X24 system is equipped with 24 channel electrodes for biopotential measurements, such as EEG, Electrocardiogram (ECG), Electromyogram (EMG), and Electrooculogram (EOG). This system measures and delivers real-time EEG signals via a Bluetooth connection. The system provides more than 8 hours of operation time and less than 10 minutes of installation time. Also, this system supports a variety of applications such as drowsiness, cognitive workload, and neuro-dynamics monitoring. 


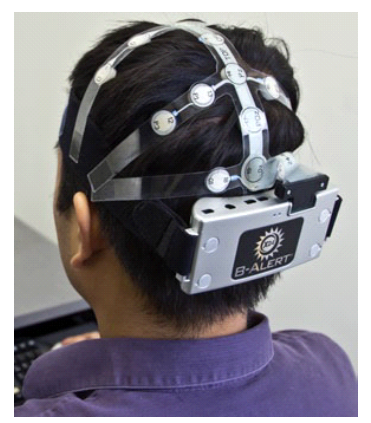

a

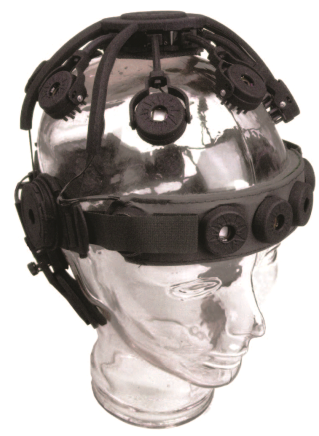

b

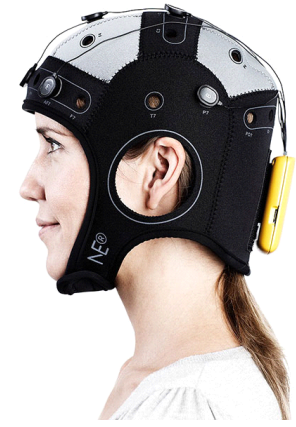

c

Figure 6. Pictures of Wireless BCI systems for research use: (a) Advanced Brain Monitoring B-Alert X24 system [52], (b) Quasar DSI 10/20 system [53], (c) Starlab Enobio system [54].

Quasar [53] has developed and released a wireless BCI solution. This solution includes Dry Sensor Interface (DSI) 10/20, wireless Data Acquisition (DAQ), and a suite of software of its own. DSI 10/20 is a wireless BCI headset which is equipped with up to 21 EEG sensors. The EEG sensors are dry electrodes and provide high input impedance good for measuring the high fidelity EEG signals. Wireless DAQ is a peripheral device for signal transmission and onboard recording using a flash memory. QStreamer is a suite of software which contains data acquisition algorithms as well as different cognitive state classification algorithms. The classification algorithms estimate user's mental states in terms of workload, engagement, and fatigue.

Enobio [54] is a wireless EEG acquisition device developed by Starlab. This device is a cap style with light weight feature (only $65 \mathrm{~g}$ ). It has multiple channels, supporting an option of 8 or 20 channels in particular; each is equipped with a dry electrode. It can operate up to 16 hours long using a rechargeable Lithium Polymer battery. It is connected with a computer via a Bluetooth connection. A bundle software provides real time visualization of EEG signals such as power spectrum and raw signal monitoring. This system has been applied to various applications associated with medical, neurofeedback, and cognitive state monitoring.

Guger technologies (G.tec) is a medical engineering company which provides comprehensive BCI solutions. This company has released a mobile biopotential acquisition system named as g.MOBIlab+ [55]. This system is available in two different modes: the 8 channel EEG acquisition mode and the multi-modal acquisition mode. In the multi-modal acquisition mode, this system can measure the EEG signals with other physiological signals such as Electrocardiogram (ECG), Electromyogram (EMG), and Electrooculogram (EOG). With a Bluetooth connection, it operates up to 100 hours using four AA batteries. 


\section{Challenges and future research directions for wireless BCI systems}

Many wireless BCI systems have already been developed for consumers or research uses, and they are attracting public attention. However, only limited areas of applications, such as medical and entertainment applications, have taken advantages of these systems so far. Wireless BCI systems have not yet fully made their way into our life. In this section, we aim to analyze the current challenges the wireless BCI systems research must face, and discuss a few possible future research directions to enable wireless BCI systems to be practically utilized in a much wider scale.

\subsection{Challenges in wireless BCI systems research}

Wireless BCI systems currently face the following problems: 1) insufficiency in features controllable by EEG signals, 2) deficiency in accuracy in EEG signal interpretation, and 3) lack of killer applications.

First, the available features of EEG signals are limited to be utilized for increasing the speed and accuracy of brain computer communications. The EEG signals are not easy for a user to freely generate in user's own intension. It means that the controllable dimension of EEG signals is not large. In the EEG-based BCI systems, sensorimotor rhythms (SMRs), visual evoked potentials (VEPs) and brain rhythms which generated by particular cognitive states are used as controllable features. Among these features, SMRs usually require long-term training to be adopted in control of $\mathrm{BCI}$ applications. Without enough training, the accuracy of SMR-based BCI is usually very low. Also, VEPs cannot be generated without visual stimulations. Therefore, among the wireless BCI systems on scientific articles and commercial products, many BCI systems have utilized the specific brain rhythms generated under the specific cognitive states of users [27][46][49][50][51], such as attention and relaxation, as controllable features of EEG signals. The users can control an application by intentionally changing their own cognitive state. For example, in [27], the users can control the direction of arrows in an archery game, using a wireless BCI system. The direction of arrows is determined from the quantification of the focusing intensity of the users. As can be seen here, the intensity level is only single dimensional; the degree of control the users can issue cannot but be limited. This limitation in the control dimension comes with easiness in adapting to a program within a short learning time, but it plays a limiting role as well. Consequently, the applications BCI systems can be applied are limited as well. To remedy this limitation, some research groups have focused on discovering other controllable EEG features. One example is to use detection of various cognitive states, such as drowsiness and alertness [20][29][30] [32]. With all these efforts, however, the number of features that can be obtained is still considered very limited. Namely, wireless BCI systems today can interpret only simple massages from user's intensions.

Second, current BCI systems are not reliable enough to be used in accuracy-critical applications, such as vehicle controls and data telecommunications. To utilize them in a wide range of applications, improving the accuracy of brain computer communication is one of most important issues. In wireless BCI systems, many features of the EEG signals, such as cogni- 
tive states, event related potentials (ERPs) in P300, and steady-state visual evoked potentials (SSVEP), are used for BCI based controls. These features are easily affected by various noise and inference sources. For example, in ambulatory applications, such as the workload monitoring [13], a user may need to wear a wireless EEG acquisition device for a long period of time and may need to be able to move around. In such a situation, the accuracy of applications can easily decline due to vibrations and noises. Furthermore, because every person has somewhat different characteristics in EEG features, training to find the best feature set should be carried out in the individual basis for achieving higher accuracy. For these reasons, most commercial wireless BCI systems are developed for less accuracy-critical applications such as computer games and home appliances.

Third, killer applications for wireless BCI systems are needed. In wireless BCI systems, a killer application can be said to be a useful application which influenced on the life of an average person. What BCI system provides ultimately is a human computer interface. But it is not the only form that human can interact with the computer. Speech recognition and hand-motion recognition are, for example, other easier means perhaps with faster and more accurate performance than a BCI can provide. In commercial wireless BCI systems, applications for game and utility control have been mostly developed for entertainment uses. In these applications, it is possible to choose an alternative control interface, such as speech recognition and hand motion, instead of the EEG signals for application controls. Thus, these applications are not a killer application. In the research field, valuable applications related to smart living environment [29][30][32], drowsiness detection [15][19][20][32], and communications [28] have been developed for wireless BCI systems, but they are still not suitable due to lack of sufficient field verifications. Therefore, identification of a killer application still remains to be an urgently needed research topic for wireless BCI systems to thrive.

\subsection{Future research directions for wireless BCI systems}

Future research on wireless BCI systems should take the following directions: 1) hybrid signal acquisition and 2) development of adaptive classification algorithms.

First, hybrid signal acquisition is needed for higher accuracy and fast brain computer interaction. Hybrid signal acquisition through simultaneous recording of multiple brain signals has been shown to ensure higher accuracy thanks to complementary analysis of user's motivations. There are two different ways to do a hybrid signal acquisition we wish to discuss here. The first is utilizing other biopotential measurements, such as Electrocardiogram (ECG), Electromyogram (EMG), and Electrooculogram (EOG). This approach is already employed in commercial wireless BCI systems such as Emotiv's EPOC system, OCZ technology's Neural Impulse Actuator, and Advanced Brain Monitoring's B-Alert X series. With these additional physiological signal measurements, the users can use not only brain waves but also facial expressions and eye movements. The second is utilizing the multiple features among the available EEG features, such as sensorimotor rhythms (SMRs), P300, and steadystate visual evoked potentials (SSVEP) simultaneously. Most commercial wireless BCI systems use only the cognitive states as the controllable feature of wireless BCI systems. If wireless BCI systems employ additional features of EEG signals which are not generated by 
the same motivation, the accuracy of the applications will be improved by adoption of the complementary classification. Recently, Brunner et al. [56] have published a paper on a hybrid BCI. In that paper, a hybrid approach using both the event-related desynchronization (ERD) and the SSVEP was experimentally found to provide better accuracy with little or no training.

Second, adaptive algorithms are needed to reduce training time and achieve higher accuracy. Because every person has a unique set of their own EEG characteristics, most applications require training procedures for learning the user's EEG patterns. However, the EEG patterns typically change continuously affected by many factors such as the mental state of the users and the circumstance surrounding the users. Furthermore, long-term training can make the users tired and induce degradation in accuracy. For these reasons, the reduction of training in applications is an important issue in BCI researches. To reduce trainings, additional signal processing schemes likes adaptive classification algorithms can be added to BCI systems. Because these schemes can discern changes in EEG features, the accuracy of applications can be improved. In the BCI research field, some researchers have already studied adaptive classification. Vidaurre et al. have published papers about adaptive discriminant analysis [57][58]. This algorithm is based on quadratic discriminant analysis (QDA) and linear discriminant analysis (LDA). Using this algorithm, they have shown that accuracy can be improved greatly in their own BCI experiments built on imagery motor movements.

\section{Conclusion}

BCI is a useful technology for people with disabilities as it can offer them an additional means of communication, and reinstate a damaged motor control function. Recently, BCI has started its way to grab the attention of the general public as well because this technology has shown the possibility of a new type of user experience. For example, drowsiness detection can be applied to car drivers for preventing traffic accidents. And, real time monitoring of bio-potential signals is useful for diagnosis of patients who have brain diseases such as epilepsy and Alzheimer's disease. To use BCI systems in real-life applications on a daily basis, portable, wearable wireless BCI systems are critical, instead of bulky and cumbersome wired BCI systems. Recently, several wireless BCI systems have been introduced by leading research groups and commercial companies.

In this book chapter, we have reviewed the recent research trends in the development of wireless BCI systems. Various research groups have focused on biosensors, user friendly system designs, and more influential applications. Also, there are a few companies which have developed and released wireless BCI systems into that market, with some commercial successes. Nevertheless, research challenges, such as the insufficiency in controllable features, the deficiency in BCI's control accuracy, and the lack of killer applications are still the issues remained to be resolved. The first two challenges are technical issues which will be resolved in time with continuous research efforts. When good wireless BCI systems which provide high-fidelity data acquisition and fast onboard signal processing are available at low cost, they will surely promote the creation of very useful real-life applications. 


\section{Acknowledgements}

This work was supported by the National Research Foundation of Korea (NRF) grant funded by the Korean government (MEST) (Do-Yak Research Program, No. 2012-0005656)

\section{Author details}

Seungchan Lee, Younghak Shin, Soogil Woo, Kiseon Kim and Heung-No Lee*

*Address all correspondence to: heungno@gist.ac.kr, seungchan@gist.ac.kr

Gwangju Institute of Science and Technology(GIST), Cheomdan-gwagiro, Buk-gu, Gwangju, Republic of Korea

\section{References}

[1] Emil S Valchinov and Nicolas E Pallikarakis, "An active electrode for biopotential recording from small localized bio-sources," BioMedical Engineering Online, Vol. 3, Iss. 1, 2004.

[2] Lun-De Liao, I-Jan Wang, Sheng-Fu Chen, Jyh-Yeong Chang and Chin-Teng Lin, “Design, Fabrication and Experimental Validation of a Novel Dry-Contact Sensor for Measuring Electroencephalography Signals without Skin Preparation," Sensors, Vol. 11, Iss. 6, pp. 5819-5834, 2011.

[3] Cristian Grozea1, Catalin D. Voinescu, and Siamac Fazli, "Bristle-sensors - Low-cost Flexible Passive Dry EEG Electrodes for Neurofeedback and BCI Applications," Journal of Neural Engineering, Vol. 8, Iss. 2, 2011.

[4] Thomas J. Sullivan, Stephen R. Deiss, and Gert Cauwenberghs, "A Low-Noise, NonContact EEG/ECG Sensor," Biomedical Circuits and Systems Conference, pp. 154 157, 2007.

[5] Yu Mike Chi, Yu-Te Wang, Yijun Wang, Christoph Maier, Tzyy-Ping Jung, and Gert Cauwenberghs, "Dry and Noncontact EEG Sensors for Mobile Brain-Computer Interfaces," IEEE Trans. on Neural Systems and Rehabilitation Engineering, Vol. 20, No. 2, 2012.

[6] Nuno Sérgio Diasa, João Paulo Carmo, Paulo Mateus Mendes, José Higino Correiac, "Wireless instrumentation system based on dry electrodes for acquiring EEG signals," Medical Engineering \& Physics, Vol. 34, pp. 972-981,

[7] Giulio Ruffini, Stephen Dunne, Esteve Farrés, Ívan Cester, Paul C. P. Watts, S. Ravi P. Silva, Carles Grau, Lluís Fuentemilla, Josep Marco-Pallarés and Bjorn Vandecasteele, 
"ENOBIO dry electrophysiology electrode; first human trial plus wireless electrode system," 29th IEEE EMBS, pp. 6689 - 6693, 2007.

[8] Gaetano Gargiulo, Rafael A. Calvo, Paolo Bifulco, Mario Cesarelli, Craig Jin, Armin Mohamed, André van Schaik, "A new EEG recording system for passive dry electrodes," Clinical Neurophysiology, Vol. 121, pp. 686-693, 2010.

[9] Gaetano Gargiulo, Paolo Bifulco, Rafael A. Calvo, Mario Cesarelli, Craig Jin and André van Schaik, "A mobile EEG system with dry electrodes," BioCAS, pp. 273 - 276, 2008.

[10] Vadim V. Nikulin, Jewgeni Kegeles, Gabriel Curio, “Miniaturized electroencephalographic scalp electrode for optimal wearing comfort," Clinical Neurophysiology, Vol. 121, Iss. 7, 2010.

[11] Robert Matthews, Neil J. McDonald, Paul Hervieux, Peter J. Turner, and Martin A. Steindorf, "A Wearable Physiological Sensor Suite for Unobtrusive Monitoring of Physiological and Cognitive State," 29th IEEE EMBS, pp. 5276 - 5281, 2007.

[12] Robert Matthews, Neil J. McDonald, Harini Anumula, Jamison Woodward, Peter J. Turner, Martin A. Steindorf, Kaichun Chang, and Joseph M. Pendleton, "Novel Hybrid Bioelectrodes for Ambulatory Zero-Prep EEG Measurements Using Multi-channel Wireless EEG System," Foundations of Augmented Cognition Lecture Notes in Computer Science, Vol. 4565, pp. 137-146, 2007.

[13] R. Matthews, P.J. Turner, N. J. McDonald, K. Ermolaev, T. Mc Manus, R.A. Shelby, and M. Steindorf, "Real Time Workload Classification from an Ambulatory Wireless EEG System Using Hybrid EEG Electrodes," 30th IEEE EMBS, pp. 5871-587, 2008.

[14] Thomas J. Sullivan, Stephen R. Deiss, Tzyy-Ping Jung, Gert Cauwenberghs, "A BrainMachine Interface Using Dry-Contact, Low-Noise EEG Sensors," IEEE ISCAS, pp. 1986-1989, 2008.

[15] Jin-Chern Chiou, Li-Wei Ko, Chin-Teng Lin, Chao-Ting Hong, Tzyy-Ping Jung, Sheng-Fu Liang and Jong-Liang Jeng, "Using Novel MEMS EEG Sensors in Detecting Drowsiness Application," IEEE BioCAS, pp. 33-36, 2006.

[16] Lindsay Brown, Jef van de Molengraft, Refet Firat Yazicioglu, Tom Torfs, Julien Penders and Chris Van Hoof, "A low-power, wireless, 8-channel EEG monitoring headset," 32nd IEEE EMBS, pp. 4197-4200, 2010.

[17] Jiawei Xu, Refet Firat Yazicioglu, Bernard Grundlehner, Pieter Harpe, Kofi A. A. Makinwa, and Chris Van Hoof, "A 160uW 8-Channel Active Electrode System for EEG Monitoring," IEEE Trans. on Biomedical Circuit and system, Vol. 5, No. 6, 2011.

[18] Lun-De Liao, I-Jan Wang, Che-Jui Chang, Bor-Shyh Lin, Chin-Teng Lin, and Kevin C. Tseng, "Human Cognitive Application by Using Wearable Mobile Brain Computer Interface," TENCON, pp. 346-351, 2010. 
[19] Chin-Teng Lin, Yu-Chieh Chen, Teng-Yi Huang, Tien-Ting Chiu, Li-Wei Ko, ShengFu Liang, Hung-Yi Hsieh, Shang-Hwa Hsu, and Jeng-Ren Duann, “Development of Wireless Brain Computer Interface with Embedded Multitask Scheduling and its Application on Real-Time Driver's Drowsiness Detection and Warning," IEEE Trans. on Biomedical Engineering, Vol. 55, No. 5, 2008.

[20] Chin-Teng Lin, Che-Jui Chang, Bor-Shyh Lin, Shao-Hang Hung, Chih-Feng Chao, and I-Jan Wang, "A Real-Time Wireless Brain-Computer Interface System for Drowsiness Detection," IEEE Trans. on Biomedical Circuit and system, pp. $214-222$, 2010.

[21] Chin-Teng Lin, Li-Wei Ko, Jin-Chern Chiou, Jeng-Ren Duann, Ruey-Song Huang, Sheng-Fu Liang, Tzai-Wen Chiu, and Tzyy-Ping Jung, "Noninvasive Neural Prostheses Using Mobile and Wireless EEG," Proceedings of the IEEE, Vol. 96, No. 7, July 2008.

[22] Chin-Teng Lin, Li-Wei Ko, Che-Jui Chang, Yu-Te Wang, Chia-Hsin Chung, Fu-Shu Yang, Jeng-Ren Duann, Tzyy-Ping Jung, and Jin-Chern Chiou, "Wearable and Wireless Brain-Computer Interface and Its Applications," HCII 2009, pp. 741-748, 2009.

[23] Thorsten Oliver Zander, Moritz Lehne, Klas Ihme, Sabine Jatzev, Joao Correia, Christian Kothe, Bernd Picht and Femke Nijboer, "A dry EEG-system for scientific research and brain-computer interfaces," Frontiers in Neuroscience, Vol. 5, 2011.

[24] Robert Lin, Ren-Guey Lee, Chwan-Lu Tseng, Yan-Fa Wu, Joe-Air Jiang, “Design and Implementation of Wireless Multi-Channel EEG Recording System and Study Of EEG Clustering Method," Biomedical Engineering applications Basis \& Communications, Vol. 18 No. 6, 2006.

[25] Luca Piccini, Sergio Parini, Luca Maggi and Giuseppe Andreoni, "A Wearable Home BCI system: preliminary results with SSVEP protocol," 27th IEEE EMBS, pp. $5384-$ 5387, 2005.

[26] Alexandre Ribeiro, António Sirgado, João Aperta, Ana Lopes, Jorge Guilherme, Pedro Correia, Gabriel Pires and Urbano Nunes, "A Low-Cost EEG Stand-Alone Device For Brain Computer Interface," BIODEVICES, pp. 430-433, 2009.

[27] Lun-De Liao, Chi-Yu Chen, I-Jan Wang, Sheng-Fu Chen, Shih-Yu Li, Bo-Wei Chen, Jyh-Yeong Chang and Chin-Teng Lin, "Gaming control using a wearable and wireless EEG-based brain-computer interface device with novel dry foam-based sensors," Journal of Neuro Engineering and Rehabilitation, Vol.9 No.5, 2012.

[28] Yu-Te Wang, Yijun Wang and Tzyy-Ping Jung, "A cell-phone-based brain-computer interface for communication in daily life," Journal of Neural Engineering, Vol.8, 2011.

[29] Chin-Teng Lin, Fu-Chang Lin, Shi-An Chen, Shao-Wei Lu, Te-Chi Chen, Li-Wei Ko, “EEG-based Brain-computer Interface for Smart Living Environmental Auto-adjust- 
ment," Journal of Medical and Biological Engineering, Vol. 30, Iss. 4, pp. 237-245, 2010 .

[30] Chin-Teng Lin, Bor-Shyh Lin, Fu-Chang Lin, and Che-Jui Chang, "Brain Computer Interface-Based Smart Living Environmental Auto-Adjustment Control System in UPnP Home Networking," IEEE Systems Journal, 2012.

[31] Guger C, Holzner C, Grönegress C, Edlinger G, Slater M, “Control of a Smart Home with a Brain-Computer Interface," 4th International Brain-Computer Interface Workshop and Training Course, 2008.

[32] Shao-Hang Hung, Che-Jui Chang, Chih-Feng Chao, I-Jan Wang, Chin-Teng Lin, Borshyh Lin, "Development of Real-time Wireless Brain Computer Interface for Drowsiness Detection," ISCAS 2010, pp. 1380-1383, 2010.

[33] Ryan C. N. D’Arcy, Sujoy Ghosh Hajra, Careesa Liu, Lauren D. Sculthorpe, and Donald F. Weaver, "Towards Brain First-Aid: A Diagnostic Device for Conscious Awareness," IEEE Transactions on Biomedical Engineering, Vol. 58, No. 3, March 2011.

[34] http://www.ti.com/product/ads1298

[35] http://www.microchip.com/pagehandler/en-us/family/16bit/architecture/pic24f.html

[36] http://www.microchip.com/pagehandler/en-us/family/16bit/architecture/ dspic33f.html

[37] http://www.ti.com/lsds/ti/microcontroller/16-bit_msp430/overview.page

[38] http://emotiv.com/store/hardware/epoc-bci/epoc-neuroheadset/

[39] Gert Pfurtschellera, Christa Neuper, "Motor imagery activates primary sensorimotor areas," Neuroscience Letters, Vol. 239, pp. 65-68, 1997.

[40] Simon P. Levine, Jane E. Huggins, Spencer L. BeMent, Ramesh K. Kushwaha, Lori A. Schuh, Mitchell M. Rohde, Erasmo A. Passaro, Donald A. Ross, Kost V. Elisevich, and Brien J. Smith, "A direct brain interface based on event-related potentials," IEEE Trans. on Rehabilitation Engineering, Vol. 8, Iss. 2, pp. 180-185, 2000.

[41] Emanuel Donchin, Kevin M. Spencer, and Ranjith Wijesinghe, "The mental prosthesis: assessing the speed of a P300-based brain-computer interface," IEEE Trans. on Rehabilitation Engineering, Vol. 8, Iss. 2, pp. 174-179, 2000.

[42] Ming Cheng, Xiaorong Gao, Shangkai Gao, and Dingfeng $\mathrm{Xu}$, "Design and implementation of a brain-computer interface with high transfer rates," IEEE Trans. on Biomedical Engineering, Vol. 49, Iss. 10, pp. 1181-1186, 2002.

[43] Carmen Vidaurre, Benjamin Blankertz, "Towards a Cure for BCI Illiteracy," Brain Topography, Vol. 23, Iss. 2, pp. 194-198, 2010. 
[44] Ling Guo, Daniel Rivero, Alejandro Pazos, "Epileptic seizure detection using multiwavelet transform based approximate entropy and artificial neural networks," Journal of Neuroscience Methods, Vol. 193, pp.156-163, 2010.

[45] F. Galán, M. Nuttinc, E. Lewa, P.W. Ferreza, G. Vanackerc, J. Philipsc, J. del R. Millán, "A brain-actuated wheelchair: Asynchronous and non-invasive Brain-computer interfaces for continuous control of robots," IEEE Trans. on Rehabilitation Engineering, Vol. 8, Iss. 4, pp. 441-446, 2000.

[46] http://www.neurosky.com/Products/MindSet.aspx

[47] http://developer.neurosky.com/docs/doku.php?id=esenses_tm

[48] http://www.neurosky.com/Products/ThinkGearAM.aspx

[49] http://www.plxdevices.com/product_info.php?id=XWAVESONIC

[50] http://myndplay.com/products.php?cat=1

[51] http://www.ocztechnology.com/nia-game-controller.html

[52] http://advancedbrainmonitoring.com/neurotechnology/systems/

[53] http://www.quasarusa.com/

[54] http://neuroelectrics.com/enobio

[55] http://www.gtec.at/Products/Hardware-and-Accessories/g.MOBIlab-Specs-Features

[56] C Brunner, B Z Allison, C Altstätter and C Neuper, "A comparison of three braincomputer interfaces based on event-related desynchronization, steady state visual evoked potentials, or a hybrid approach using both signals," Journal of Neural Engineering, Vol. 8, 2011.

[57] C. Vidaurre, A. Schlögl, R. Cabeza, R. Scherer, and G. Pfurtscheller, “A Fully On-Line Adaptive BCI," IEEE Transactions on Biomedical Engineering, Vol. 53, No. 6, 2006.

[58] C. Vidaurre, A. Schlögl, R. Cabeza, R. Scherer, and G. Pfurtscheller, "Study of OnLine Adaptive Discriminant Analysis for EEG-Based Brain Computer Interfaces," IEEE Transactions on Biomedical Engineering, Vol. 54, No. 3, 2007. 